\title{
The maximal Hilbert transform along nonconvex curves
}

Honghai Liu*

\section{"Correspondence:}

hhliu@hpu.edu.cn

School of Mathematics and

Information Science, Henan

Polytechnic University, Jiaozuo,

Henan 454003, P.R. China

\begin{abstract}
The Hilbert transform along curves is defined by the principal value integral. The pointwise existence of the principal value Hilbert transform can be educed from the appropriate estimates for the corresponding maximal Hilbert transform. By using the estimates of Fourier transforms and Littlewood-Paley theory, we obtain

$L^{P}$-boundedness for the maximal Hilbert transform associated to curves $(t, P(\gamma(t)))$, where $1<p<\infty, P$ is a real polynomial and $\gamma$ is convex on $[0, \infty)$. Then, we can conclude that the Hilbert transform along curves $(t, P(\gamma(t)))$ exists in pointwise sense. MSC: Primary 42B20; secondary 42B25
\end{abstract}

Keywords: the maximal Hilbert transform; maximal functions; nonconvex curves; Littlewood-Paley theory

\section{Introduction}

For $n \geq 2$, let $\Gamma: \mathbb{R} \rightarrow \mathbb{R}^{n}$ be a curve in $\mathbb{R}^{n}$ with $\Gamma(0)=0$. To $\Gamma$ we associate the Hilbert transform $\mathcal{H}$ which is defined as a principal value integral

$$
\mathcal{H} f(u)=\text { p.v. } \int_{\mathbb{R}} f(u-\Gamma(t)) \frac{d t}{t},
$$

where $u \in \mathbb{R}^{n}$ and $f \in C_{0}^{\infty}\left(\mathbb{R}^{n}\right)$. Similarly, one can define the corresponding maximal function and the maximal Hilbert transform as

$$
\mathcal{M} f(u)=\sup _{h>0} \frac{1}{h} \int_{-h}^{h}|f(u-\Gamma(t))| d t
$$

and

$$
\mathcal{H}^{*} f(u)=\sup _{\varepsilon>0}\left|\int_{|t| \geq \varepsilon} f(u-\Gamma(t)) \frac{d t}{t}\right| .
$$

The $L^{p}$-boundedness for the Hilbert transform $\mathcal{H}$ and the maximal function $\mathcal{M}$ above have been well studied by many scholars. See [10] for a survey of results through 1977. More recent results can be found in [1, 2, 4-7].

Appropriate estimates for the maximal Hilbert transform give the pointwise existence of the principal value Hilbert transform. So, we focus on the $L^{p}$-bounds for the maximal Hilbert transform $\mathcal{H}^{*}$ in this paper. Let us state some previous theorems to establish

\section{㩏 Springer}

(c) 2012 Liu; licensee Springer. This is an Open Access article distributed under the terms of the Creative Commons Attribution License (http://creativecommons.org/licenses/by/2.0), which permits unrestricted use, distribution, and reproduction in any medium, provided the original work is properly cited. 
the background for our current work. The first result about $\mathcal{H}^{*}$ is the work of Stein and Wainger (see [10]).

\section{Theorem 1.1}

(A) If $\Gamma$ is a two-sided homogeneous curve in $\mathbb{R}^{n}$, then

$$
\left\|\mathcal{H}^{*} f\right\|_{L^{p}} \leq C\|f\|_{L^{p}}, \quad 1<p<\infty .
$$

(B) Assume that for small $t, \Gamma(t)$ lies in the subspace spanned by $\left\{\Gamma^{(j)}(0)\right\}_{j=1}^{\infty}$. Then the maximal Hilbert transform

$$
\mathcal{H}_{1}^{*} f(u)=\sup _{0<\varepsilon \leq 1}\left|\int_{\varepsilon \leq|t| \leq 1} f(u-\Gamma(t)) \frac{d t}{t}\right|
$$

is bounded from $L^{p}\left(\mathbb{R}^{n}\right)$ to itself, $1<p<\infty$.

For $n=2$ and $\Gamma(t)=(t, \gamma(t))$, Córdoba and Rubio de Francia considered the case $\gamma \in$ $C^{1}(\mathbb{R})$ and $\gamma(0)=\gamma^{\prime}(0)=0$, with the following properties:

(i) $\gamma$ is biconvex, i.e., $\left|\gamma^{\prime}(t)\right|$ is decreasing in $(-\infty, 0)$ and increasing in $(0, \infty)$;

(ii) $\left|\gamma^{\prime}\right|$ has doubling time, i.e., there exists a constant $\lambda>1$ such that $\left|\gamma^{\prime}(\lambda t)\right| \geq 2\left|\gamma^{\prime}(t)\right|$;

(iii) $\gamma$ is balanced, by which we mean the following: there exists $k>1$ such that

$$
\left|\gamma\left(k^{-1} t\right)\right| \leq|\gamma(-t)| \leq|\gamma(k t)| \text { for every } t>0 .
$$

They proved the following theorem in [7].

Theorem 1.2 Under the assumptions (i), (ii) and (iii) on the $\gamma$, the maximal Hilbert transform $\mathcal{H}^{*}$ is a bounded operator in $L^{p}\left(\mathbb{R}^{2}\right)$ for $1<p<\infty$.

In this note, we consider the curve $\Gamma$ with the form $\Gamma(t)=(t, P(\gamma(t)))$, where $P(t)$ is a real-valued polynomial of $t$ in $\mathbb{R}, \gamma$ satisfies

$$
\gamma \in C^{2}[0, \infty), \quad \text { convex on }[0, \infty) \text {, even or odd on } \mathbb{R} \text {, and } \gamma(0)=0 \text {. }
$$

Definition 1.3 A function $f: \mathbb{R} \rightarrow \mathbb{R}$ belongs to $\mathcal{C}_{1}$, if there exists a constant $\lambda>1$ such that $f(\lambda t)>2 f(t)$ for $t \in \mathbb{R}$. It is also said that $f$ has doubling time.

For this case, Bez obtained the $L^{p}$-boundedness of $\mathcal{H}$ and $\mathcal{M}$ in [1].

Theorem 1.4 Suppose that $P$ is a polynomial, $\gamma$ satisfies (1.1) and $\gamma^{\prime}(0) \geq 0$. If $\Gamma(t)=$ $(t, P(\gamma(t))), 1<p<\infty$, and either (1) $P^{\prime}(0)$ is zero, or (2) $P^{\prime}(0)$ is nonzero and $\gamma^{\prime} \in \mathcal{C}_{1}$, then

$$
\|\mathcal{H} f\|_{L^{p}} \leq C\|f\|_{L^{p}} \quad \text { and } \quad\|\mathcal{M} f\|_{L^{p}} \leq C\|f\|_{L^{p}}
$$

Moreover, the constant $C$ depends only on $p, \gamma$ and the degree of $P$.

Remark 1.5 If $P^{\prime}(0)=0, P(\gamma(t))$ is "more convex" than $\gamma(t)$ in some sense, then $\Gamma$ is "convex" enough for the $L^{p}$-boundedness of $\mathcal{M}$ and $\mathcal{H}$. In the case $P^{\prime}(0) \neq 0$, the linear term $P^{\prime}(0) t$ of $P(t)$ cannot improve the convexity of $\gamma$. To obtain the $L^{p}$-boundedness for asso- 
ciated operators, one needs to pose additional condition(s) on $\gamma$, that is, $\gamma^{\prime} \in \mathcal{C}_{1}$. For more details, see [5] and [9].

Motivated by Bez's result above, we obtain the $L^{p}$-boundedness for $\mathcal{H}^{*}$. More precisely, we prove the following theorem.

Theorem 1.6 Suppose that $P$ is a polynomial, $\gamma$ satisfies (1.1) and $\gamma^{\prime}(0) \geq 0$. If $\Gamma(t)=$ $(t, P(\gamma(t)))$, and either $(1) P^{\prime}(0)$ is zero, or (2) $P^{\prime}(0)$ is nonzero and $\gamma^{\prime} \in \mathcal{C}_{1}$, then

$$
\left\|\mathcal{H}^{*} f\right\|_{L^{p}} \leq C\|f\|_{L^{p}}, \quad 1<p<\infty .
$$

Moreover, the constant $C$ depends only on $p, \gamma$ and the degree of $P$.

Remark 1.7 Let $P(t)=t$. Comparing those conditions for $\gamma$ in Theorem 1.2, we find that conditions in Theorem 1.6 are stricter. But we should note that $P(\gamma(t))$ may be a nonconvex function.

The convexity of the polynomial $P$ is important for our main result. $P$ has different convexity in different intervals, which suggests that $\mathcal{H}^{*}$ will be decomposed according to the properties of $P$. The decay of associated multipliers is essential for the proof of Theorem 1.6. This set of techniques originated from the work [1] and [3]. Notice that $\mathcal{H}^{*}$ is a nonlinear operator, Minkowski's inequality cannot be used as in Section 1 of [1], the linearization method is invoked to treat it. Similarly, the essential Proposition 1.2 in [1] is useless for the maximal Hilbert transform. Littlewood-Paley theory and interpolation theorem are effective tools to treat those problems. Those ideas are due to the contribution of Córdoba, Nagel, Vance, Wainger, Rubio de Francia.

The organization of our paper is as follows. In Section 2, we list some key properties concerning the polynomial and give some lemmas for the proof of the main result. The $L^{p}$-estimates for $\mathcal{H}^{*}$ will be proved in Section 3.

\section{Preliminaries}

Without loss of generality, we suppose that $P(t)=\sum_{k=1}^{d} p_{k} t^{k}$, where $d \geq 2$. Let $z_{1}, z_{2}, \ldots, z_{d}$ be $d$-complex roots of $P$ ordered as

$$
0=\left|z_{1}\right| \leq\left|z_{2}\right| \leq \cdots \leq\left|z_{d}\right|
$$

Let $A$ be a positive constant which will be chosen in Lemma 2.1. Define $G_{j}=\left(A\left|z_{j}\right|\right.$, $\left.A^{-1}\left|z_{j+1}\right|\right]$ if it is nonempty for $1 \leq j<d$ and $G_{d}=\left(A\left|z_{d}\right|, \infty\right)$. Let $\mathcal{J}=\left\{j: G_{j} \neq \emptyset\right\}$, then $(0, \infty) \backslash \bigcup_{j \in \mathcal{J}} G_{j}$ can be decomposed as $\bigcup_{k \in \mathcal{K}} D_{k}$, where $D_{k}$ is the interval between two adjacent $G_{j}$. It is obvious that $D_{k}$ is disjoint. Then, we can decompose $(0, \infty)$ as

$$
(0, \infty)=\bigcup_{j \in \mathcal{J}} \gamma^{-1}\left(G_{j}\right) \cup \bigcup_{k \in \mathcal{K}} \gamma^{-1}\left(D_{k}\right)
$$

where $\gamma^{-1}(I)$ is the inverse image of a subset $I$ restricted in $(0, \infty)$.

The properties of $P$ on $D_{k}$ and $G_{j}$ are important for our proof. The following related lemma can be found in [1] and [3]. 
Lemma 2.1 There exists a number $C_{d}>1$ such that for any $A \geq C_{d}$ and any $j \in \mathcal{J}$,

(i) $|P(t)| \sim\left|p_{j}\right||t|^{j}$ for $|t| \in G_{j}$;

(ii) $\frac{P^{\prime}(t)}{P(t)}>0$ for $t \in G_{j}, \frac{P^{\prime}(t)}{P(t)}<0$ for $-t \in G_{j}$;

(iii) $\left|\frac{P^{\prime}(t)}{P(t)}\right| \sim \frac{1}{|t|}$ for $|t| \in G_{j}$;

(iv) $\frac{P^{\prime \prime}(t)}{P(t)}>0$ and $\frac{P^{\prime \prime}(t)}{P(t)} \sim \frac{1}{t^{2}}$ for $|t| \in G_{j}, j \in \mathcal{J} \backslash\{1\}$.

The following fact can be induced from the proof of Lemma 2.1 (see [1]), that is, we can choose $A>0$ such that, for $|t| \in G_{j}$,

$$
|P(t)| \leq 2\left|p_{j}\right||t|^{j} \quad \text { and } \quad \frac{1}{2} j\left|p_{j}\right||t|^{j-1} \leq\left|P^{\prime}(t)\right| \leq 2 j\left|p_{j}\right||t|^{j-1} .
$$

Let $\lambda$ be the doubling constant for $\gamma^{\prime}$, define $\rho=\max \{3, \lambda\}$. Let $I$ be a subset of $(0, \infty)$, $x, s \in \mathbb{R}$ and $u=(x, s), \mathcal{H}_{I}$ and $\mathcal{M}_{I}$ are given by

$$
\mathcal{H}_{I} f(u)=\int_{|t| \in \gamma^{-1}(I)} f(x-t, s-P(\gamma(t))) \frac{d t}{t},
$$

and

$$
\mathcal{M}_{I} f(u)=\sup _{k \in \mathbb{Z}} \frac{1}{\rho^{k}} \int_{|t| \in \gamma^{-1}(I) \cap\left(\rho^{k}, \rho^{k+1}\right]}|f(x-t, s-P(\gamma(t)))| d t .
$$

For $j \in \mathcal{J}$ and $k \in \mathbb{Z}$, we define cones in $\mathbb{R}^{2}$ by

$$
\Delta_{k, j}=\left\{\zeta=(\xi, \eta): \frac{1}{4}\left|p_{j}\right|\left(\gamma^{j}\right)^{\prime}\left(\rho^{k}\right)<\frac{|\xi|}{|\eta|}<4\left|p_{j}\right|\left(\gamma^{j}\right)^{\prime}\left(\rho^{k+1}\right), \xi, \eta \in \mathbb{R}\right\}
$$

and the corresponding projection operators by $\widehat{T_{k, j}} f=\widehat{f} \cdot \chi_{\Delta_{k, j}}$. Then, we have the following lemma which is Lemma 1 in [7].

Lemma 2.2 For $j \in \mathcal{J}$ and $1<p<\infty$, we have

(i) $\left\|\left(\sum_{k \in \mathbb{Z}}\left|T_{k, j} f\right|^{2}\right)^{\frac{1}{2}}\right\|_{L^{p}} \leq C_{p}\|f\|_{L^{p}}$;

(ii) $\left\|\sum_{k \in \mathbb{Z}} T_{k, j} f_{k}\right\|_{L^{p}} \leq C_{p}\left\|\left(\sum_{k \in \mathbb{Z}}\left|f_{k}\right|^{2}\right)^{\frac{1}{2}}\right\|_{L^{p}}$;

(iii) $\left\|\sup _{i \in \mathbb{Z}} \mid \sum_{k \geq i} T_{k, f} f\right\|_{L^{p}} \leq C_{p}\|f\|_{L^{p}}$.

The bootstrap argument (see [8]) plays an important role in the proof of the main result, so we present the following well-known result which can be found in [2] and [7].

Lemma 2.3 Suppose that $U_{k} f=u_{k} * f$ is a sequence of positive operators uniformly bounded in $L^{\infty}$, and $U^{*} f=\sup _{k \in \mathbb{Z}}\left|u_{k} * f\right|$ is bounded in $L^{q}$ for $1<q<\infty$, then

$$
\left\|\left(\sum_{k \in \mathbb{Z}}\left|U_{k} f_{k}\right|^{2}\right)^{\frac{1}{2}}\right\|_{L^{p}} \leq C_{p}\left\|\left(\sum_{k \in \mathbb{Z}}\left|f_{k}\right|^{2}\right)^{\frac{1}{2}}\right\|_{L^{p}}
$$

for $p>\frac{2 q}{1+q}$. 


\section{Proof of Theorem 1.6}

Let $\mathcal{K}$ and $\mathcal{J}$ be given as in Section 2, then

$$
\begin{aligned}
\mathcal{H}^{*} f(u) \leq & \sum_{k \in \mathcal{K}} \sup _{\varepsilon>0}\left|\int_{|t| \in \gamma^{-1}\left(D_{k}\right) \cap\{t \geq \varepsilon\}} f(x-t, s-P(\gamma(t))) \frac{d t}{t}\right| \\
& +\sum_{j \in \mathcal{J}} \sup _{\varepsilon>0}\left|\int_{|t| \in \gamma^{-1}\left(G_{j}\right) \cap\{t \geq \varepsilon\}} f(x-t, s-P(\gamma(t))) \frac{d t}{t}\right| \\
:= & \sum_{k \in \mathcal{K}} \mathcal{H}_{D_{k}}^{*} f(u)+\sum_{j \in \mathcal{J}} \mathcal{H}_{G j}^{*} f(u) .
\end{aligned}
$$

Note that $\mathcal{K}$ and $\mathcal{J}$ are finite sets, it suffices to show that $\mathcal{H}_{D_{k}}^{*}$ and $\mathcal{H}_{G_{j}}^{*}$ are $L^{p}$-bounded, respectively.

\subsection{The $L^{p}$-boundedness for $\mathcal{H}_{D_{k}}^{*}$}

Let $\varepsilon(u)$ be some measurable function from $\mathbb{R}^{2}$ to $\mathbb{R}^{+}$such that

$$
\mathcal{H}_{D_{k}}^{*} f(u) \leq 2\left|\int_{|t| \in \gamma^{-1}\left(D_{k}\right) \cap\{t \geq \varepsilon(u)\}} f(x-t, s-P(\gamma(t))) \frac{d t}{t}\right| .
$$

By Minkowski's inequality, we can control the $L^{p}$-norm of $\mathcal{H}_{D_{k}}^{*} f$ by

$$
\begin{aligned}
& \left(\int_{\mathbb{R}^{2}}\left|\int_{|t| \in \gamma^{-1}\left(D_{k}\right) \cap\{t \geq \varepsilon(u)\}} f(x-t, s-P(\gamma(t))) \frac{d t}{t}\right|^{p} d u\right)^{\frac{1}{p}} \\
& \quad \leq \int_{|t| \in \gamma^{-1}\left(D_{k}\right)}\left(\int_{\mathbb{R}^{2}}|f(x-t, s-P(\gamma(t)))|^{p} d u\right)^{\frac{1}{p}} \frac{d t}{|t|} \\
& \quad \leq 2\|f\|_{L^{p}} \int_{\gamma^{-1}\left(D_{k}\right)} \frac{d t}{t} .
\end{aligned}
$$

Let $D_{k}=\left(A^{-1}\left|z_{j}\right|, A\left|z_{j+m}\right|\right]$ for some $2 \leq j \leq d$ and $0 \leq m \leq d-j$, then

$$
A^{-1}\left|z_{j}\right| \leq A^{-1}\left|z_{j+1}\right| \leq A\left|z_{j}\right| \leq \cdots \leq A\left|z_{j+m}\right|<A^{-1}\left|z_{j+m+1}\right|
$$

and

$$
A^{2} \leq \frac{A\left|z_{j+m}\right|}{A^{-1}\left|z_{j}\right|} \leq \frac{A\left|z_{j+m}\right|}{A^{-2 m}\left|z_{j+m}\right|} \leq A^{2 m+1} .
$$

Notice that $\gamma$ is convex and $\gamma(0)=0$, so $\gamma(t) \leq t \gamma^{\prime}(t)$ for $t>0$. Thus,

$$
\begin{aligned}
\int_{\gamma^{-1}\left(D_{k}\right)} \frac{d t}{t} & =\int_{\gamma^{-1}\left(A^{-1}\left|z_{j}\right|\right)}^{\gamma^{-1}\left(A\left|z_{j+m}\right|\right)} \frac{d t}{t}=\int_{A^{-1}\left|z_{j}\right|}^{A\left|z_{j+m}\right|} \frac{d t}{\gamma^{-1}(t) \gamma^{\prime}\left(\gamma^{-1}(t)\right)} \\
& \leq \int_{A^{-1}\left|z_{j}\right|}^{A\left|z_{j+m}\right|} \frac{d t}{t} \leq 2 d \ln A,
\end{aligned}
$$

where $\gamma^{-1}(t)$ is the inverse function of $\gamma(t)$. 
(3.1), (3.2) and (3.3) yield

$$
\left\|\mathcal{H}_{D_{k}}^{*} f\right\|_{L^{p}} \leq C\|f\|_{L^{p}} \quad \text { for } 1<p<\infty, k \in \mathcal{K} .
$$

\subsection{The $L^{p}$-boundedness for $\mathcal{H}_{G_{j}}^{*}$}

For $j \in \mathcal{J}$ and $k \in \mathbb{Z}$, set $I_{k, j}=(1, \rho] \cap \rho^{-k} \gamma^{-1}\left(G_{j}\right)$ and define measures $v_{k, j}$ by

$$
\left\langle v_{k, j}, \psi\right\rangle=\int_{|t| \in I_{k, j}} \psi\left(\rho^{k} t, P\left(\gamma\left(\rho^{k} t\right)\right)\right) \frac{d t}{t}
$$

for $\psi \in \mathcal{S}\left(\mathbb{R}^{2}\right)$. For any $\varepsilon>0$, there exists $k_{0} \in \mathbb{Z}$ such that $\rho^{k_{0}} \leq \varepsilon<\rho^{k_{0}+1}$. Then

$$
\begin{aligned}
& \left|\int_{|t| \in \gamma^{-1}\left(G_{j}\right) \cap\{t \geq \varepsilon\}} f(x-t, s-P(\gamma(t))) \frac{d t}{t}\right| \\
& \leq \frac{1}{\rho^{k_{0}}} \int_{|t| \in \gamma^{-1}\left(G_{j}\right) \cap\left(\rho^{\left.k_{0}, \rho^{k_{0}+1}\right]}\right.}|f(x-t, s-P(\gamma(t)))| d t \\
& \quad+\left|\sum_{k=k_{0}+1}^{\infty} v_{k, j} * f(u)\right| .
\end{aligned}
$$

Therefore,

$$
\mathcal{H}_{G, j}^{*} f(u) \leq \mathcal{M}_{G_{j}} f(u)+\sup _{i \in \mathbb{Z}}\left|\sum_{k=i}^{\infty} v_{k, j} * f(u)\right| .
$$

By the $L^{p}$-boundedness of $\mathcal{M}_{G_{j}}$ (see [1]), it suffices to consider the latter term. Let $\Phi \in$ $\mathcal{S}(\mathbb{R})$ such that $\widehat{\Phi}(\xi)=1$ for $|\xi| \leq 1$ and $\widehat{\Phi}(\xi)=0$ for $|\xi| \geq 2$. Write $\widehat{\Phi}_{i}(\xi)=\widehat{\Phi}\left(\rho^{i} \xi\right)$ and denote by $\star$ convolution in the first variable. For $i \in \mathbb{Z}$, the truncated Hilbert transform can be decomposed as

$$
\begin{aligned}
\sum_{k \geq i} v_{k, j} * f= & \Phi_{i} \star\left(\mathcal{H}_{G_{j}} f-\sum_{k<i} v_{k, j} * f\right)+\left(\delta-\Phi_{i}\right) \star \sum_{k \geq i} v_{k, j} * T_{k, j} f \\
& +\left(\delta-\Phi_{i}\right) \star \sum_{k \geq i} v_{k, j} *\left(\mathcal{I}-T_{k, j}\right) f=: \mathcal{A}_{i, j} f+\mathcal{B}_{i, j} f+\mathcal{C}_{i, j} f,
\end{aligned}
$$

where $\delta$ is the Dirac measure in $\mathbb{R}$, and $\mathcal{I}$ is the identity operator. Then, we just need to estimate $\sup _{i \in \mathbb{Z}}\left|\mathcal{A}_{i, j} f\right| \sup _{i \in \mathbb{Z}}\left|\mathcal{B}_{i, f} f\right|$ and $\sup _{i \in \mathbb{Z}}\left|\mathcal{C}_{i, j} f\right|$, respectively.

The decay of $\widehat{v_{k, j}}(\zeta)$ is important for the boundedness of three maximal operators above. Essentially, estimates for $\widehat{v_{k, j}}$ in the following subsection have been proved in [1]. We repeat them just for completeness.

\subsubsection{Fourier transform estimates of $v_{k, j}$}

Before the proof of Proposition 3.2, we need the following lemma which is Lemma 2.2 in [1].

Lemma 3.1 For all $j \in \mathcal{J} \backslash\{1\}$, the function

$$
t \mapsto P^{\prime \prime}\left(\gamma\left(\rho^{k} t\right)\right) \gamma^{\prime}\left(\rho^{k} t\right)^{2}+P^{\prime}\left(\gamma\left(\rho^{k} t\right)\right) \gamma^{\prime \prime}\left(\rho^{k} t\right)
$$

is singled-signed on $I_{k, j}$. 
Proposition 3.2 For $j \in \mathcal{J}$ and $k \in \mathbb{Z}$, if $\zeta \notin \Delta_{k, j}$, then

$$
\left|\widehat{v_{k, j}}(\zeta)\right| \leq C\left(\rho^{k}|\xi|\right)^{-1}
$$

Proof For fixed $\zeta=(\xi, \eta)$, let $h_{k}(t)=\rho^{k} t \xi+\eta P\left(\gamma\left(\rho^{k} t\right)\right)$, then

$$
\left|\widehat{v_{k, j}}(\zeta)\right| \leq\left|\int_{t \in I_{k, j}} e^{-i h_{k}(t)} \frac{d t}{t}\right|+\left|\int_{t \in-I_{k, j}} e^{-i h_{k}(t)} \frac{d t}{t}\right|:=E(\xi, \eta)+F(\xi, \eta) .
$$

Case 1. $j \in \mathcal{J} \backslash\{1\}$. If $\zeta \notin \Delta_{k, j}$ and $|\xi|>4\left|p_{j}\right|\left(\gamma^{j}\right)^{\prime}\left(\rho^{k+1}\right)|\eta|$, for $|t| \in I_{k, j}$, (2.1) implies

$$
\left|h_{k}^{\prime}(t)\right| \geq\left|\rho^{k} \xi\right|-\left|\rho^{k} P^{\prime}\left(\gamma\left(\rho^{k} t\right)\right) \gamma^{\prime}\left(\rho^{k} t\right) \eta\right| \geq \frac{1}{2} \rho^{k}|\xi| .
$$

Note that $h_{k}^{\prime}(t)$ is monotone on $I_{k, j}$, this fact follows from Lemma 3.1. By Van der Corput's lemma and (3.4), we get $E(\xi, \eta) \leq C\left(\rho^{k}|\xi|\right)^{-1}$.

If $\gamma$ is even, then $F(-\xi, \eta)=E(\xi, \eta)$. If $\gamma$ is odd, Lemma 3.1 still holds for $t \in-I_{k, j}, F(\xi, \eta)$ can be considered in the same way. Then, we have

$$
\left|\widehat{v_{k, j}}(\zeta)\right| \leq E(\xi, \eta)+F(\xi, \eta) \leq C\left(\rho^{k}|\xi|\right)^{-1}
$$

If $\zeta \notin \Delta_{k, j}$ and $|\xi|<\frac{1}{4}\left|p_{j}\right|\left(\gamma^{j}\right)^{\prime}\left(\rho^{k}\right)|\eta|$. In the same way, for $|t| \in I_{k, j}$, we have

$$
\left|h_{k}^{\prime}(t)\right| \geq\left|\rho^{k} P^{\prime}\left(\gamma\left(\rho^{k} t\right)\right) \gamma^{\prime}\left(\rho^{k} t\right) \eta\right|-\left|\rho^{k} \xi\right| \geq \frac{1}{4}\left|p_{j}\right|\left(\gamma^{j}\right)^{\prime}\left(\rho^{k} t\right) \rho^{k}|\eta| \geq C \rho^{k}|\xi| .
$$

In the same way as above, we can get

$$
\left|\widehat{v_{k, j}}(\zeta)\right| \leq C\left(\rho^{k}|\xi|\right)^{-1}
$$

Case 2. $j=1$. If $\zeta \notin \Delta_{k, 1}$ and $|\xi|>4\left|p_{1}\right| \gamma^{\prime}\left(\rho^{k+1}\right)|\eta|$, (3.4) still holds for $|t| \in I_{k, 1}$. By integrating by parts,

$$
\begin{aligned}
E(\xi, \eta) & =\left|\int_{I_{k, 1}} e^{-i h_{k}(t)} h_{k}^{\prime}(t) \frac{d t}{h_{k}^{\prime}(t) t}\right| \\
& \leq 2\left(\rho^{k}|\xi|\right)^{-1}+\int_{I_{k, 1}} \frac{\left|h_{k}^{\prime}(t)+t h_{k}^{\prime \prime}(t)\right|}{\left[t h_{k}^{\prime}(t)\right]^{2}} d t \\
& \leq C\left(\rho^{k}|\xi|\right)^{-1}+\int_{I_{k, 1}} \frac{\left|h_{k}^{\prime \prime}(t)\right|}{h_{k}^{\prime}(t)^{2}} d t .
\end{aligned}
$$

Essentially, we just need to consider the second term, which can be dominated by

$$
\int_{I_{k, 1}} \frac{\rho^{2 k}|\eta|\left|P^{\prime}\left(\gamma\left(\rho^{k} t\right)\right)\right| \gamma^{\prime \prime}\left(\rho^{k} t\right)}{h_{k}^{\prime}(t)^{2}} d t+\int_{I_{k, 1}} \frac{\rho^{2 k}\left|\eta \| P^{\prime \prime}\left(\gamma\left(\rho^{k} t\right)\right)\right| \gamma^{\prime}\left(\rho^{k} t\right)^{2}}{h_{k}^{\prime}(t)^{2}} d t:=\alpha_{1}+\alpha_{2} .
$$

In order to estimate the term $\alpha_{1}$, we define $\varphi_{k}(t)=\rho^{k} t|\xi|+\left|p_{1}\right| \gamma\left(\rho^{k} t\right)|\eta|$, then $\varphi_{k}^{\prime}(t)=$ $\rho^{k}|\xi|+\left|p_{1}\right| \rho^{k} \gamma^{\prime}\left(\rho^{k} t\right)|\eta|$. By (3.4), for $t \in I_{k, 1}$, it is obvious that

$$
\left|\varphi_{k}^{\prime}(t)\right| \leq \frac{5}{4} \rho^{k}|\xi| \leq C h_{k}^{\prime}(t)
$$


On the other hand, for $t \in I_{k, 1}$,

$$
\left|\varphi_{k}^{\prime}(t)\right| \geq \rho^{k}|\xi|-\left|p_{1}\right| \rho^{k} \gamma^{\prime}\left(\rho^{k} t\right)|\eta| \geq \frac{3}{4} \rho^{k}|\xi|
$$

Further, by (2.1), for $t \in I_{k, 1}$,

$$
\varphi_{k}^{\prime \prime}(t)=\left|p_{1}\right| \rho^{2 k} \gamma^{\prime \prime}\left(\rho^{k} t\right)|\eta| \geq \frac{1}{2} \rho^{2 k}|\eta|\left|P^{\prime}\left(\gamma\left(\rho^{k} t\right)\right)\right| \gamma^{\prime \prime}\left(\rho^{k} t\right) .
$$

Thus, combining (3.7), (3.9) and (3.8), we have

$$
\alpha_{1} \leq C \int_{I_{k, 1}} \frac{\varphi_{k}^{\prime \prime}(t)}{\varphi_{k}^{\prime}(t)^{2}} d t \leq C\left(\rho^{k}|\xi|\right)^{-1}
$$

For $\alpha_{2}$, by (3.4),

$$
\begin{aligned}
\alpha_{2} & \leq C \int_{I_{k, 1}}\left|P^{\prime \prime}\left(\gamma\left(\rho^{k} t\right)\right)\right| \gamma^{\prime}\left(\rho^{k} t\right) \rho^{k} \frac{\rho^{k}|\eta| \gamma^{\prime}\left(\rho^{k+1}\right)}{h_{k}^{\prime}(t)^{2}} d t \\
& \leq C\left(\left|p_{1}\right| \rho^{k}|\xi|\right)^{-1} \int_{G_{1}}\left|P^{\prime \prime}(t)\right| d t .
\end{aligned}
$$

Note that $G_{1}$ can be split into a finite number of disjoint intervals such that $P^{\prime \prime}$ is singledsigned on each interval. Suppose that $\left[s_{1}, s_{2}\right]$ is such an interval and $P^{\prime \prime} \geq 0$ by $(2.1)$, then $\int_{\left[s_{1}, s_{2}\right]}\left|P^{\prime \prime}(t)\right| d t=P^{\prime}\left(s_{2}\right)-P^{\prime}\left(s_{1}\right) \leq C\left|p_{1}\right|$. So $\alpha_{2} \leq C\left(\rho^{k}|\xi|\right)^{-1}$.

If $(\xi, \eta) \notin \Delta_{k, 1}$ and $|\xi| \leq \frac{1}{4}\left|p_{1}\right| \gamma^{\prime}\left(\rho^{k}\right)|\eta|$, (3.6) holds for $|t| \in I_{k, 1}$. The same arguments used above imply

$$
E(\xi, \eta) \leq C\left(\left|p_{1}\right| \rho^{k} \gamma^{\prime}\left(\rho^{k}\right)|\eta|\right)^{-1}+\int_{I_{k, 1}} \frac{\left|h_{k}^{\prime \prime}(t)\right|}{h_{k}^{\prime}(t)^{2}} d t \leq C\left(\rho^{k}|\xi|\right)^{-1}+\alpha_{1}+\alpha_{2},
$$

where $\alpha_{1}$ and $\alpha_{2}$ are as previous ones. For $\varphi_{k}$ above, we have

$$
\frac{3}{4}\left|p_{1}\right| \rho^{k} \gamma^{\prime}\left(\rho^{k} t\right)|\eta| \leq\left|\varphi_{k}^{\prime}(t)\right| \leq \frac{5}{4}\left|p_{1}\right| \gamma^{\prime}\left(\rho^{k} t\right) \rho^{k}|\eta| \leq 5 h_{k}^{\prime}(t) .
$$

Thus, (3.9) and (3.12) give

$$
\alpha_{1} \leq C \int_{I_{k, 1}} \frac{\varphi_{k}^{\prime \prime}(t)}{\varphi_{k}^{\prime}(t)^{2}} d t \leq C\left(\left|p_{1}\right| \rho^{k} \gamma^{\prime}\left(\rho^{k}\right)|\eta|\right)^{-1} \leq C\left(\rho^{k}|\xi|\right)^{-1}
$$

For $\alpha_{2}$, by (3.12) and (2.1),

$$
\begin{aligned}
\alpha_{2} & \leq C \int_{I_{k, 1}} \frac{\rho^{2 k}|\eta|\left|P^{\prime \prime}\left(\gamma\left(\rho^{k} t\right)\right)\right| \gamma^{\prime}\left(\rho^{k} t\right)^{2}}{\left[\left|p_{1}\right| \rho^{k} \gamma^{\prime}\left(\rho^{k} t\right)|\eta|\right]^{2}} d t \\
& =C \int_{I_{k, 1}}\left|p_{1}\right|^{-1} \rho^{k}\left|P^{\prime \prime}\left(\gamma\left(\rho^{k} t\right)\right)\right| \gamma^{\prime}\left(\rho^{k} t\right) \frac{1}{\left|p_{1}\right| \rho^{k} \gamma^{\prime}\left(\rho^{k} t\right)|\eta|} d t \\
& \leq C\left(\left|p_{1}\right| \rho^{k} \gamma^{\prime}\left(\rho^{k}\right)|\eta|\right)^{-1} \int_{G_{1}}\left|p_{1}\right|^{-1}\left|P^{\prime \prime}(t)\right| d t \\
& \leq C\left(\rho^{k}|\xi|\right)^{-1} .
\end{aligned}
$$


$F(\xi, \eta)$ can be treated in the same way as in Case 1. Thus, (3.10), (3.11), (3.13) and (3.14) imply

$$
\left|\widehat{v_{k, j}}(\xi, \eta)\right| \leq C\left(\rho^{k}|\xi|\right)^{-1} .
$$

3.2.2 $L^{p}$-estimates for $\sup _{i \in \mathbb{Z}}\left|\mathcal{A}_{i, j} f\right|$

3.2.2.1 The case of even $\gamma$ By a linear transformation, we have

$$
\begin{aligned}
\Phi_{i} & \star \sum_{k<i} v_{k, j} * f(u) \\
& =\sum_{k<i} \int_{|t| \in \rho^{k} I_{k, j}} \frac{1}{t} \int_{\mathbb{R}} \Phi_{i}(x-y-t) f(y, s-P(\gamma(t))) d y d t \\
& =\sum_{k<i} \int_{|t| \in \rho^{k} I_{k, j}} \frac{1}{t} \int_{\mathbb{R}}\left[\Phi_{i}(x-y-t)-\Phi_{i}(x-y)\right] f(y, s-P(\gamma(t))) d y d t .
\end{aligned}
$$

Note that $\Phi \in \mathcal{S}$, then for any $N>0$,

$$
\begin{aligned}
& \left|\Phi_{i} \star \sum_{k<i} v_{k, j} * f(u)\right| \\
& \quad \leq \int_{|t| \in\left(0, \rho^{i}\right] \cap \gamma^{-1}\left(G_{j}\right)} \frac{1}{|t|} \int_{\mathbb{R}} \frac{|t| \rho^{-i}}{\rho^{i}\left(1+\rho^{-i}|x-y|\right)^{N}}|f(y, s-P(\gamma(t)))| d y d t \\
& \quad \leq \int_{\mathbb{R}} \frac{\rho^{-i}}{\left(1+\left|\rho^{-i} x-\rho^{-i} y\right|\right)^{N}} \frac{1}{\rho^{i}} \int_{|t| \in\left(0, \rho^{i}\right] \cap \gamma^{-1}\left(G_{j}\right)}|f(y, s-P(\gamma(t)))| d t d y \\
& \quad \leq C\left(\mathcal{N}_{j} f\right)^{\star}(u),
\end{aligned}
$$

where $f^{\star}(x, s)$ is the Hardy-Littlewood maximal function acting on $f(y, s)$ in the first variable and $\mathcal{N}_{j}$ is given by

$$
\mathcal{N}_{j} g(s)=\sup _{i \in \mathbb{Z}} \frac{1}{\rho^{i}} \int_{t \in\left(0, \rho^{i}\right] \cap \gamma^{-1}\left(G_{j}\right)}|g(s-P(\gamma(t)))| d t .
$$

Thus, we obtain

$$
\sup _{i \in \mathbb{Z}}\left|\mathcal{A}_{i, j} f(u)\right| \leq C\left[\left(\mathcal{N}_{j} f\right)^{\star}(u)+\left(\mathcal{H}_{G_{j}} f\right)^{\star}(u)\right] .
$$

If we can show that $\mathcal{N}_{j}$ is $L^{p}$-bounded, according to the $L^{p}$-boundedness of $\mathcal{H}_{G_{j}}$ (see [1]), we will get

$$
\left\|\sup _{i \in \mathbb{Z}}\left|\mathcal{A}_{i, j} f\right|\right\|_{L^{p}} \leq C\|f\|_{L^{p}}, \quad 1<p<\infty .
$$

So, it suffices to prove the following result.

Lemma 3.3 For $j \in \mathcal{J}, \mathcal{N}_{j}$ is a bounded operator in $L^{p}(\mathbb{R}), 1<p<\infty$.

Proof We denote $P(\gamma(t))$ by $\Upsilon(t)$ for short, then $\Upsilon(t)^{\prime}=P^{\prime}(\gamma(t)) \gamma^{\prime}(t)$. Note that there is no root of $P(s)$ in $G_{j}$, that is, $P(s)$ is singled-signed. For $t \in \gamma^{-1}\left(G_{j}\right), \gamma(t) \in G_{j}$, by (2) of 
Lemma 2.1, $P^{\prime}(\gamma(t))$ is also singled-signed on $\gamma^{-1}\left(G_{j}\right)$. By $\gamma^{\prime}(0) \geq 0$ and the convexity of $\gamma$, $\gamma^{\prime}(t)>0$ for $t>0$. Then, $\Upsilon(t)$ is monotonous on $\gamma^{-1}\left(G_{j}\right)$. On the other hand, by Lemma 3.1, for $j \in \mathcal{J} \backslash\{1\}, \Upsilon(t)^{\prime}$ is monotonous on $\gamma^{-1}\left(G_{j}\right)$.

Suppose that $\Upsilon(t)$ is increasing on $\gamma^{-1}\left(G_{j}\right)$, then

$$
\begin{aligned}
& \frac{1}{\rho^{i}} \int_{t \in\left(0, \rho^{i}\right] \cap \gamma^{-1}\left(G_{j}\right)}|g(s-\Upsilon(t))| d t=\frac{1}{\rho^{i}} \int_{t \in\left(0, \Upsilon\left(\rho^{i}\right)\right] \cap P\left(G_{j}\right)}|g(s-t)| \frac{d t}{\Upsilon^{\prime}\left(\Upsilon^{-1}(t)\right)} \\
&:=\int_{0}^{\infty}|g(s-t)| \phi_{i, j}(t) d t .
\end{aligned}
$$

For $j \in \mathcal{J} \backslash\{1\}$, if $\Upsilon^{\prime}(t)$ is increasing on $\gamma^{-1}\left(G_{j}\right)$, then, for $i \in \mathbb{Z}, \phi_{i, j}(t)$ is nonnegative and decreasing on $P\left(G_{j}\right)$. Furthermore, note that

$$
\int_{0}^{\infty} \phi_{i, j}(t) d t \leq \frac{1}{\rho^{i}} \int_{t \in\left(0, \Upsilon\left(\rho^{i}\right)\right]} \frac{d t}{\Upsilon^{\prime}\left(\Upsilon^{-1}(t)\right)}=1
$$

Therefore, for $i \in \mathbb{Z}$, we have

$$
\frac{1}{\rho^{i}} \int_{t \in\left(0, \rho^{i}\right] \cap \gamma^{-1}\left(G_{j}\right)}|g(s-\Upsilon(t))| d t \leq C M g(s),
$$

where $M$ is the Hardy-Littlewood maximal function.

If $\Upsilon^{\prime}(t)$ is decreasing on $\gamma^{-1}\left(G_{j}\right)$, write

$$
\int_{0}^{\infty}|g(s-t)| \phi_{i, j}(t) d t=\int_{0}^{\infty}|\tilde{g}(-s+t)| \widetilde{\phi_{i, j}}(-t) d t=\int_{-\infty}^{0}|\tilde{g}(-s-t)| \widetilde{\phi_{i, j}}(t) d t
$$

where $\tilde{g}$ denotes the reflection of $g$. Notice that $\widetilde{\phi_{i, j}}(t)$ is nonnegative and decreasing on $-P\left(G_{j}\right)$ and $\left\|\widetilde{\phi_{i, j}}\right\|_{L^{1}} \leq 1$. Similarly,

$$
\frac{1}{\rho^{i}} \int_{t \in\left(0, \rho^{i}\right] \cap \gamma^{-1}\left(G_{j}\right)}|g(s-\Upsilon(t))| d t \leq C M \tilde{g}(-s) .
$$

For $j=1, \Upsilon(t)$ and $\gamma(t)$ are increasing on $\gamma^{-1}\left(G_{1}\right)$ and $\mathbb{R}^{+}$, respectively. Then, $P(t)$ is increasing on $G_{1}$, that is, $P^{\prime}(t)>0$. According to (2.1), $\frac{1}{2}\left|p_{1}\right| \leq P^{\prime}(t) \leq 2\left|p_{1}\right|$; furthermore, $\frac{1}{2}\left|p_{1}\right| t \leq P(t) \leq 2\left|p_{1}\right| t$ for $t \in G_{1}$. Therefore, combining the convexity of $\gamma$, we have

$$
\begin{aligned}
& \frac{1}{\rho^{i}} \int_{t \in\left(0, \Upsilon\left(\rho^{i}\right) \cap \cap P\left(G_{1}\right)\right.}|g(s-t)| \frac{d t}{\Upsilon^{\prime}\left(\Upsilon^{-1}(t)\right)} \\
& \quad \leq \frac{1}{\rho^{i}} \int_{t \in\left(0,2\left|p_{1}\right| \gamma\left(\rho^{i}\right)\right] \cap 2\left|p_{1}\right| G_{1}}|g(s-t)| \frac{d t}{\frac{1}{2}\left|p_{1}\right| \gamma^{\prime}\left(\gamma^{-1}\left(2\left|p_{1}\right|^{-1} t\right)\right)} \\
& \quad \leq \frac{1}{\rho^{i}} \int_{t \in\left(0,4 \gamma\left(\rho^{i}\right)\right) \cap 4 G_{1}}\left|g\left(s-\frac{t\left|p_{1}\right|}{2}\right)\right| \frac{d t}{\gamma^{\prime}\left(\gamma^{-1}(t)\right)} \leq C M g_{\frac{\left|p_{1}\right|}{2}}\left(\frac{2}{\left|p_{1}\right|} s\right),
\end{aligned}
$$

where $g_{\frac{\left|p_{1}\right|}{2}}(t)=g\left(\frac{\left|p_{1}\right| t}{2}\right)$.

By the $L^{p}$-boundedness of $M$, we complete the proof of Lemma 3.3. 
3.2.2.2 The case of odd $\gamma$ We decompose $v_{k, j}$ as $v_{k, j}=\widetilde{v_{k, j}}+m_{k, j}$, where $\widehat{m_{k, j}}(\xi, \eta)=$ $\widehat{\Phi}\left(\rho^{k} \xi\right) \widehat{\nu_{k, j}}(0, \eta)$. Therefore,

$$
\left|\Phi_{i} \star \sum_{k<i} v_{k, j} * f(u)\right| \leq\left|\Phi_{i} \star \sum_{k<i} \widetilde{v_{k, j}} * f(u)\right|+\left|\Phi_{i} \star \sum_{k<i} m_{k, j} * f(u)\right|
$$

For $\Phi_{i} \star \sum_{k<i} \widetilde{v_{k, j}} * f$, note that

$$
\Phi_{i} \star \widetilde{\nu_{k, j}} * f(u)=\int_{|t| \in \rho^{k} I_{k, j}} \frac{1}{t} \int_{\mathbb{R}}\left[\Phi_{i}(x-y-t)-\Phi_{i} * \Phi_{k}(x-y)\right] f(y, s-P(\gamma(t))) d y d t .
$$

For $k<i, \widehat{\Phi}_{i}=\widehat{\Phi}_{i} \cdot \widehat{\Phi_{k}}$, we have

$$
\int_{\mathbb{R}}\left[\Phi_{i}(x-y)-\Phi_{i} * \Phi_{k}(x-y)\right] f(y, s-P(\gamma(t))) d y=0 .
$$

Similar to the case of even $\gamma$, we obtain

$$
\sup _{i \in \mathbb{Z}}\left|\Phi_{i} \star \sum_{k<i} \widetilde{\nu_{k, j}} * f(u)\right| \leq C\left(\mathcal{N}_{j} f\right)^{\star}(u)
$$

For the second term in the right-hand side of (3.16),

$$
\sup _{i \in \mathbb{Z}}\left|\Phi_{i} \star \sum_{k<i} m_{k, j} * f(u)\right| \leq C\left(\sup _{i \in \mathbb{Z}}\left|\sum_{k<i} m_{k, j} * f\right|\right)^{\star}(u)
$$

Then, we get the estimate

$$
\sup _{i \in \mathbb{Z}}\left|\mathcal{A}_{i, j} f(u)\right| \leq C\left[\left(\mathcal{N}_{j} f\right)^{\star}(u)+\left(\sup _{i \in \mathbb{Z}}\left|\sum_{k<i} m_{k, j} * f\right|\right)^{\star}(u)+\left(\mathcal{H}_{G_{j}} f\right)^{\star}(u)\right]
$$

By Lemma 3.3 and the $L^{p}$-boundedness for $\mathcal{H}_{G_{j}}$, we just need to prove that

$$
\left\|\sup _{i \in \mathbb{Z}}\left|\sum_{k<i} m_{k, j} * f\right|\right\|_{L^{p}} \leq C\|f\|_{L^{p}}, \quad 1<p<\infty
$$

To obtain (3.18), we denote the set $\left\{(\xi, \eta):\left[\left|p_{j}\right| \gamma^{j}\left(\rho^{k+1}\right)\right]^{-1} \leq|\eta| \leq\left[\left|p_{j}\right| \gamma^{j}\left(\rho^{k}\right)\right]^{-1}\right\}$ by $L_{k, j}$ and define projection operators $S_{k, j}$ by $\widehat{S_{k, j} f}=\widehat{f} \cdot \chi_{L_{k, j}}$. Then, we use the following majorization:

$$
\sup _{i \in \mathbb{Z}}\left|\sum_{k<i} m_{k, j} * f\right| \leq \sum_{l \in \mathbb{Z}} \sup _{i \in \mathbb{Z}}\left|\sum_{k<i} m_{k, j} * S_{l+k} f\right| \leq \sum_{l \in \mathbb{Z}} E_{l, j} f
$$

where $E_{l, j} f=\left(\sum_{k \in \mathbb{Z}}\left|m_{k, j} * S_{l+k} f\right|^{2}\right)^{\frac{1}{2}}$.

We will prove that for $j \in \mathcal{J}$ and $l \in \mathbb{Z}$,

$$
\left\|E_{l, j} f\right\|_{L^{p}} \leq C\|f\|_{L^{p}}, \quad 1<p<\infty,
$$


and

$$
\left\|E_{l, j} f\right\|_{L^{2}} \leq C \rho^{-|l|}\|f\|_{L^{2}} .
$$

(3.19) and interpolation between (3.20) and (3.21) give (3.18).

To prove (3.20), we use the fact $\sup _{k \in \mathbb{Z}}\left|m_{k, j} * f(u)\right| \leq C\left(\mathcal{N}_{j} f\right)^{\star}(u)$, Lemma 3.3 and Lemma 2.3,

$$
\left\|E_{l, j} f\right\|_{L^{p}}=\left\|\left(\sum_{k \in \mathbb{Z}}\left|m_{k, j} * S_{l+k} f\right|^{2}\right)^{\frac{1}{2}}\right\|_{L^{p}} \leq C\left\|\left(\sum_{k \in \mathbb{Z}}\left|S_{l+k} f\right|^{2}\right)^{\frac{1}{2}}\right\|_{L^{p}} \leq C\|f\|_{L^{p}} .
$$

For (3.21), by Plancherel theorem,

$$
\left\|E_{l, j} f\right\|_{L^{2}}^{2}=\sum_{k \in \mathbb{Z}} \int_{L_{l+k, j}}|\widehat{f}(\zeta)|^{2}\left|\widehat{m_{k, j}}(\zeta)\right|^{2} d \zeta
$$

So, it suffices to show

$$
\left|\widehat{m_{k, j}}(\zeta)\right| \leq \rho^{-|l|} \text { for } \zeta \in L_{l+k, j} \text { and } l \in \mathbb{Z}
$$

By (2.1), we have

$$
\left|\widehat{v_{k, j}}(0, \eta)\right|=\left|\int_{|t| \in I_{k, j}}\left(e^{-i \eta P\left(\gamma\left(\rho^{k} t\right)\right)}-1\right) \frac{d t}{t}\right| \leq C\left|p_{j}\right||\eta| \gamma^{j}\left(\rho^{k+1}\right) .
$$

On the other hand, by (2.1) and the convexity of $\gamma$,

$$
\left|P\left(\gamma\left(\rho^{k} t\right)\right)^{\prime}\right| \geq \frac{1}{2}\left|p_{j}\right| j \gamma^{j-1}\left(\rho^{k} t\right) \gamma^{\prime}\left(\rho^{k} t\right) \rho^{k} \geq \frac{1}{2}\left|p_{j}\right| \gamma^{j}\left(\rho^{k}\right) .
$$

By the argument similar to that in the proof of Proposition 3.2, we obtain

$$
\left|\widehat{v_{k, j}}(0, \eta)\right| \leq C\left[|\eta|\left|p_{j}\right| \gamma^{j}\left(\rho^{k}\right)\right]^{-1}
$$

Notice that $|\widehat{\Phi}(\zeta)| \leq 1$. (3.23) and (3.24) imply

$$
\left|\widehat{m_{k, j}}(\zeta)\right| \leq C \min \left\{\left[\left|p_{j}\right||\eta| \gamma^{j}\left(\rho^{k}\right)\right]^{-1},\left|p_{j}\right||\eta| \gamma^{j}\left(\rho^{k+1}\right)\right\}
$$

For $\zeta \in L_{l+k, j}$, by (3.25) and the convexity of $\gamma$, we have

$$
\left|\widehat{m_{k, j}}(\zeta)\right| \leq\left|p_{j}\right||\eta| \gamma^{j}\left(\rho^{k+1}\right) \leq C \frac{\gamma^{j}\left(\rho^{k+1}\right)}{\gamma^{j}\left(\rho^{k+l}\right)} \leq C \rho^{-l}, \quad l \geq 1 ;
$$

and

$$
\left|\widehat{m_{k, j}}(\zeta)\right| \leq C\left[\left|p_{j}\right||\eta| \gamma^{j}\left(\rho^{k}\right)\right]^{-1} \leq C \frac{\gamma^{j}\left(\rho^{k+l+1}\right)}{\gamma^{j}\left(\rho^{k}\right)} \leq C \rho^{l}, \quad l<0 .
$$

This ends the proof of (3.22). 


\subsection{3 $L^{p}$-estimates for $\sup _{i \in \mathbb{Z}}\left|\mathcal{B}_{i, j} f\right|$}

For fixed $j \in \mathcal{J}, \sum_{k \in \mathbb{Z}} \chi_{\Delta_{k, j}}=C_{j}$, where $C_{j}$ are positive integers less than 5 . Then

$$
\sum_{k \geq i} v_{k, j} * T_{k, j} f=C_{j}^{-1} \sum_{k \geq i} T_{k, j}\left(\sum_{l \in \mathbb{Z}} v_{l, j} * T_{l, j} f\right):=C_{j}^{-1} \sum_{k \geq i} T_{k, j} \mathcal{H}_{G, j}^{I} f .
$$

Therefore,

$$
\sup _{i \in \mathbb{Z}}\left|\mathcal{B}_{i, j} f\right| \leq C \sup _{i \in \mathbb{Z}}\left|\left(\delta-\Phi_{i}\right) \star \sum_{k \geq i} T_{k, j} \mathcal{H}_{G j}^{I} f\right| \leq C\left(\sup _{i \in \mathbb{Z}}\left|\sum_{k \geq i} T_{k, j} \mathcal{H}_{G j}^{I} f\right|\right)^{\star} .
$$

For operators $\mathcal{H}_{G_{j}}^{I}$, by Lemma 2.2 and Lemma 2.3, we have

$$
\begin{aligned}
\left\|\mathcal{H}_{G, j}^{I} f\right\|_{L^{p}} & =\left\|\sum_{l \in \mathbb{Z}} T_{l, j} * v_{l, j} * T_{l, j} f\right\|_{L^{p}} \leq C_{p}\left\|\left(\sum_{l \in \mathbb{Z}}\left|v_{l, j} * T_{l, j} f\right|^{2}\right)^{1 / 2}\right\|_{L^{p}} \\
\leq & C_{p}\left\|\left(\sum_{l \in \mathbb{Z}}\left|T_{l, j} f\right|^{2}\right)^{1 / 2}\right\|_{L^{p}} \leq C_{p}\|f\|_{L^{p}},
\end{aligned}
$$

where we have used the fact that $\sup _{l \in \mathbb{Z}}\left|v_{l, j} * f\right| \leq \mathcal{M}_{G j} f$.

Finally, Lemma 2.2, (3.26) and (3.27) give

$$
\left\|\sup _{i \in \mathbb{Z}}\left|\mathcal{B}_{i, j} f\right|\right\|_{L^{p}} \leq C\|f\|_{L^{p}}, \quad 1<p<\infty
$$

3.2.4 $L^{p}$-estimates for $\sup _{i \in \mathbb{Z}}\left|\mathcal{C}_{i, j} f\right|$

The last term can be decomposed as

$$
\sup _{i \in \mathbb{Z}}\left|\mathcal{C}_{i, j} f\right|=\sup _{i \in \mathbb{Z}}\left|\left(\delta-\Phi_{i}\right) \star \sum_{k=0}^{\infty} v_{k+i, j} *\left(\mathcal{I}-T_{k+i, j}\right) f\right| \leq \sum_{k=0}^{\infty} \mathcal{P}_{k, j} f
$$

where

$$
\mathcal{P}_{k, j} f=\sup _{i \in \mathbb{Z}}\left|\left(\delta-\Phi_{i}\right) \star v_{k+i, j} *\left(\mathcal{I}-T_{k+i, j}\right) f\right|
$$

Set $T_{j}^{*} f=\sup _{k \in \mathbb{Z}}\left|T_{k, j} f\right|$, then, $\mathcal{P}_{k, j} f \leq C\left(\mathcal{M}_{G_{j}} f+\mathcal{M}_{G_{j}} T_{j}^{*} f\right)^{\star}$. By the $L^{p}$-boundedness of $\mathcal{M}_{G_{j}}$ and Lemma 2.2, we obtain

$$
\left\|\mathcal{P}_{k, j} f\right\|_{L^{p}} \leq C\|f\|_{L^{p}}, \quad 1<p<\infty .
$$

On the other hand, for $p=2$, Plancherel theorem and Proposition 3.2 imply

$$
\begin{aligned}
\left\|\mathcal{P}_{k, j} f\right\|_{L^{2}}^{2} & \leq \sum_{i \in \mathbb{Z}} \int_{\zeta \notin \Delta_{k+i, j}}\left|1-\widehat{\Phi}\left(\rho^{i} \xi\right)\right|^{2}\left|\widehat{v_{k+i, j}}(\zeta)\right|^{2}|\widehat{f}(\zeta)|^{2} d \zeta \\
& \leq C \int_{\mathbb{R}^{2}} \sum_{i: 1 \leq \rho^{i}|\xi|}\left|\rho^{i+k} \xi\right|^{-2}|\widehat{f}(\zeta)|^{2} d \zeta \\
& \leq C \rho^{-2 k}\|f\|_{L^{2}}^{2} .
\end{aligned}
$$


Interpolation between (3.29)-(3.30) and (3.28) gives

$$
\left\|\sup _{i \in \mathbb{Z}}\left|\mathcal{C}_{i, j} f\right|\right\|_{L^{p}} \leq C\|f\|_{L^{p}}, \quad 1<p<\infty
$$

\section{Competing interests}

The authors declare that they have no competing interests.

\section{Acknowledgements}

The author was supported by Doctor Foundation of Henan Polytechnic University (Grant: B2011-034).

Received: 11 March 2012 Accepted: 17 August 2012 Published: 31 August 2012

\section{References}

1. Bez, N: $L^{p}$-boundedness for the Hilbert transform and maximal operator along a class of nonconvex curves. Proc. Am. Math. Soc. 135, 151-161 (2007)

2. Carbery, A, Christ, M, Vance, J, Wainger, S, Watson, D: Operators associated to flat plane curves: $L^{p}$ estimates via dilation methods. Duke Math. J. 59, 675-700 (1989)

3. Carbery, A, Ricci, F, Wright, J: Maximal functions and Hilbert transforms associated to polynomials. Rev. Mat. Iberoam. 14, 117-144 (1998)

4. Carbery, A, Vance, J, Wainger, S, Watson, D: The Hilbert transform and maximal function along flat curves, dilations, and differential equations. Am. J. Math. 116, 1203-1239 (1994)

5. Carleson, H, Christ, M, Córdoba, A, Duoandikoetxea, J, Rubio de Francia, JL, Vance, J, Wainger, S, Weinberg, D: $L^{p}$ estimates for maximal functions and Hilbert transforms along flat convex curves in $\mathbb{R}^{2}$. Bull. Am. Math. Soc. 14, 263-267 (1986)

6. Córdoba, A, Nagel, A, Vance, J, Wainger, S, Weinberg, D: $L^{p}$ bounds for Hilbert transforms along convex curves. Invent. Math. 83, 59-71 (1986)

7. Córdoba, A, Rubio de Francia, JL: Estimates for Wainger's singular integrals along curves. Rev. Mat. Iberoam. 2, 105-117 (1986)

8. Nagel, A, Stein, EM, Wainger, S: Differentiation in lacunary directions. Proc. Natl. Acad. Sci. USA 75, 1060-1062 (1978)

9. Nagel, A, Vance, J, Wainger, S, Weinberg, D: Hilbert transforms for convex curves. Duke Math. J. 50, 735-744 (1983)

10. Stein, EM, Wainger, S: Problems in harmonic analysis related to curvature. Bull. Am. Math. Soc. 84, 1239-1295 (1978)

doi:10.1186/1029-242X-2012-191

Cite this article as: Liu: The maximal Hilbert transform along nonconvex curves. Journal of Inequalities and Applications 2012 2012:191.

\section{Submit your manuscript to a SpringerOpen ${ }^{\circ}$ journal and benefit from:}

- Convenient online submission

Rigorous peer review

- Immediate publication on acceptance

Open access: articles freely available online

- High visibility within the field

- Retaining the copyright to your article 